\title{
Diagnóstico inesperado en paciente con sospecha de GIST: infiltración gástrica por mieloma múltiple
}

\author{
Unexpected diagnosis in a patient with suspected GIST: gastric infiltration due to multiple \\ myeloma
}

Laura Martí-Gelonch*, José I. Asensio-Gallego, Emma Eizaguirre-Letamendia, Francisco J. Murgoitio-Lazkano, Íñigo Arana-Iñiguez y José M. Enríquez-Navascués

Servicio de Cirugía General y Digestiva, Sección de Cirugía Esofagogástrica y Obesidad, Hospital Universitario Donostia, San Sebastián, España

\begin{abstract}
Resumen
El mieloma múltiple se caracteriza por la proliferación neoplásica medular de células plasmáticas productoras de inmunoglobulina monoclonal. Un porcentaje pequeño de pacientes presenta compromiso extramedular en forma de plasmocitoma, siendo la localización más habitual las vías respiratorias altas. La afectación gastrointestinal es rara y la clínica asociada dependerá de la localización, la extensión y el mecanismo de infiltración. La afectación gástrica en forma de tumoración tiene un aspecto y una sintomatología similares a los de otras lesiones, por lo que es necesario realizar un diagnóstico histológico para un adecuado tratamiento. A continuación se presenta el caso de un mieloma múltiple con afectación gástrica.
\end{abstract}

Palabras clave: Estómago. GIST. Hemorragia. Mieloma.

\begin{abstract}
Multiple myeloma is characterized by medullary neoplastic proliferation of plasma cells producing monoclonal immunoglobulin. A small percentage of patients have extramedullary involvement in form of plasmacytoma, the most common location being the upper respiratory tract. Gastrointestinal involvement is rare and the associated symptoms will depend on the location, extent and mechanism of infiltration. Gastric involvement presents an appearance and symptoms similar to other lesions, so a histological diagnosis is necessary for proper treatment. This article presents the case of multiple myeloma with gastric involvement.
\end{abstract}

Key words: Stomach. GIST. Hemorrhage. Mieloma.

\section{Introducción}

El mieloma múltiple se caracteriza por la proliferación neoplásica de células plasmáticas productoras de inmunoglobulina (lg) monoclonal. Aunque la mayoría de los pacientes presentan únicamente compromiso intramedular, hasta en un $20 \%$ puede haber compromiso extramedular en forma de plasmocitoma, siendo la localización más habitual las vías respiratorias altas (orofaringe, nasofaringe, senos paranasales y laringe) ${ }^{1}$. La afectación del tracto digestivo es rara; representa

\section{Correspondencia:}

*Laura Martí Gelonch

Paseo Dr. Beguiristain, 109

Fecha de recepción: 29-05-2020

C.P. 20014, San Sebastián, Guipúzcoa, España $\quad$ Fecha de aceptación: 19-07-2020

E-mail: laura.martigelonch@osakidetza.eus

DOI: $10.24875 / C I R U .20000566$

0009-7411/@ 2020 Academia Mexicana de Cirugía. Publicado por Permanyer. Este es un artículo open access bajo la licencia CC BY-NC-ND (http://creativecommons.org/licenses/by-nc-nd/4.0/).
Cir Cir. 2020;88(S2):90-93

Contents available at PubMed

www.cirugiaycirujanos.com 
menos del $5 \%$ de los plasmocitomas y menos del $1 \%$ de los casos de mieloma múltiple ${ }^{2}$. La localización gastrointestinal más habitual es el intestino delgado, seguido del estómago, el colon y el esófago'. La mayoría de los pacientes con afectación gastrointestinal son diagnosticados durante el seguimiento de la enfermedad o en recidivas, siendo excepcional su diagnóstico como primera manifestación. A continuación se presenta el caso de un plasmocitoma gástrico que debuta con hemorragia digestiva, y se realiza una revisión de la literatura.

\section{Caso clínico}

Mujer de 76 años con antecedentes de hipertensión y diabetes tipo 2, diagnosticada de mieloma múltiple IgG-kappa en febrero de 2018, en estadio IIIA ISS-3, con afectación únicamente intramedular con múltiples lesiones óseas y tratada con nueve ciclos de bortezomib-ciclofosfamida-dexametasona (VCD) con muy buena respuesta parcial en diciembre de 2018. En seguimiento en consultas de hematología, en el último control, en diciembre de 2019, presenta estabilidad clínica pero con una lenta progresión de la gammapatía, por lo que se plantea reiniciar el tratamiento antimieloma.

Previo al inicio del tratamiento, ingresa en el servicio de digestivo en enero de 2020 por melenas junto con anemización hasta una hemoglobina de 7,9, sin presentar clínica digestiva ni repercusión hemodinámica. Se inicia el estudio realizando una gastroscopia preferente (Fig. 1), con hallazgo de una lesión de aspecto submucoso en la curvatura mayor de unos $50 \mathrm{~mm}$ de diámetro, con gran úlcera central que sugiere tumoración del estroma gastrointestinal (GIST, gastrointestinal stromal tumor); se realizan biopsias de la lesión. Se completa el estudio con tomografía computarizada toracoabdominopélvica (Fig. 2), en la que se objetiva, además de la masa en el fundus gástrico de $7 \times 2,6 \mathrm{~cm}$, un nódulo sólido paravertebral izquierdo de $2,4 \mathrm{~cm}$ y múltiples lesiones óseas líticas. Se realiza también una resonancia magnética de cuerpo entero solicitada por el servicio de hematología, y se objetivan los mismos hallazgos que en la tomografía computarizada.

Se presenta el caso en el comité de tumores y se decide realizar cirugía resectiva de la lesión gástrica, sospechosa de GIST, por hemorragia digestiva, a pesar de no tener los resultados de anatomía patológica y previo a reiniciar el tratamiento del mieloma. Antes de la fecha programada para la intervención, la

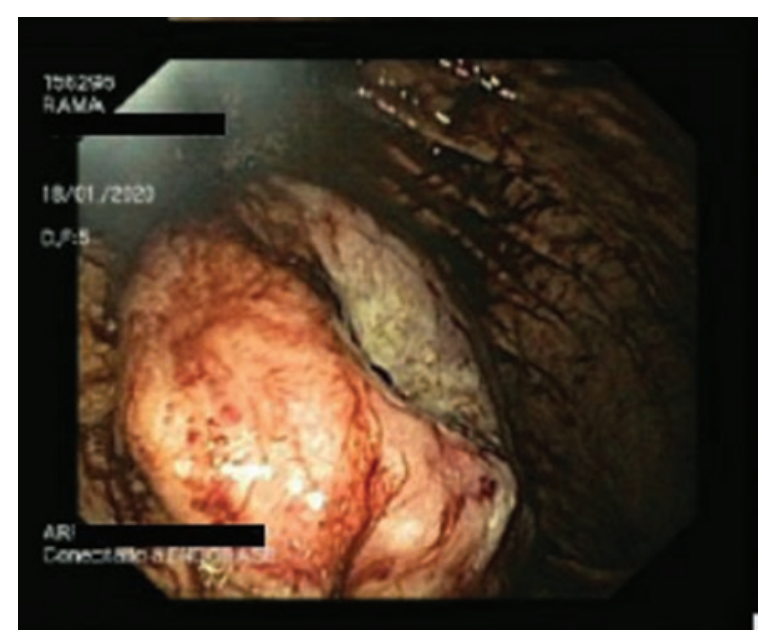

Figura 1. Imagen endoscópica de la lesión.

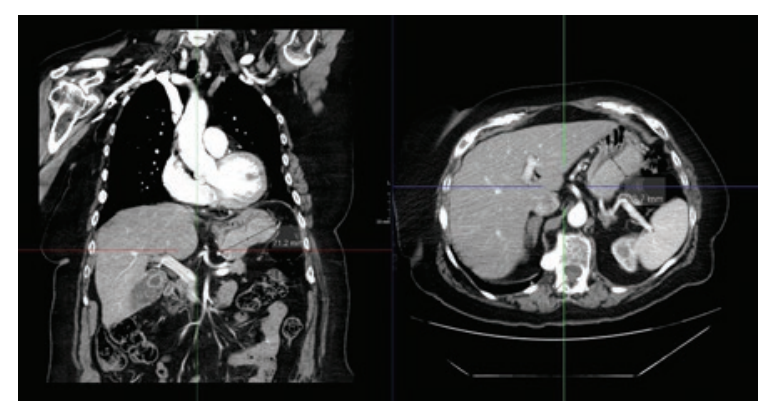

Figura 2. Imagen de tomografía computarizada de la lesión.

paciente reingresa por melenas y anemización hasta una hemoglobina de 4,8, por lo que se decide realizar una cirugía preferente.

Se interviene a principios de febrero de 2020 mediante abordaje laparoscópico, que muestra una tumoración de gran tamaño en el cuerpo gástrico, cara posterior, de unos $12 \mathrm{~cm}$ de diámetro máximo, umbilicada y muy friable. Presenta adenopatías aumentadas de tamaño en el tronco celíaco. Dado que se desconoce todavía la naturaleza de la lesión, y ante los hallazgos intraoperatorios sospechosos de malignidad, se decide realizar una gastrectomía total con linfadenectomía D2 y reconstrucción en Y de Roux.

El posoperatorio transcurre sin incidencias y se reinicia la dieta oral al séptimo día, tras realizar un estudio baritado que no objetivaba fugas. Al décimo día de posoperatorio presenta fiebre y dolor en el hipocondiro derecho secundario a un hematoma intraabdominal infectado que requiere drenaje percutáneo, con mejoría posterior. La paciente es dada de alta en el día 21 de posoperatorio. 
El estudio anatomopatológico de la pieza reveló una tumoración de $12,5 \times 9 \mathrm{~cm}$ correspondiente a una proliferación neoplásica de células plasmáticas con restricción de cadenas ligeras Kappa. Cinco ganglios linfáticos del grupo 1 presentaban proliferación de células plasmáticas; el resto eran reactivos. Se hallaron además tres implantes en el tejido adiposo del tronco celíaco. Las biopsias realizadas preoperatoriamente también mostraban infiltración gástrica por mieloma de células plasmáticas kappa.

Actualmente la paciente se encuentra a la espera de iniciar el tratamiento antimieloma.

\section{Discusión}

El plasmocitoma extramedular es poco frecuente, puede aparecer de forma solitaria o asociado a mieloma múltiple, representando una progresión extramedular de la enfermedad ${ }^{3}$. La localización más habitual es las vías aéreas altas, y la afectación gastrointestinal rara. En un estudio publicado por Talamo, et al. ${ }^{2}$ en el que se revisaban 2584 casos de mieloma múltiple, solo $24(0,9 \%)$ presentaban afectación gastrointestinal. Además, se observó que la afectación gástrica al manifestarse la enfermedad es mucho más infrecuente que cuando se presenta en el curso de una enfermedad avanzada o en progresión, como en nuestra paciente, asociándose en estos casos a mal pronóstico.

La sintomatología asociada a la afectación gastrointestinal depende de la extensión y se produce por tres mecanismos: invasión directa de un órgano, efecto masa 0 ascitis mielomatosa ${ }^{2}$. Cuando afecta al estómago, el mecanismo suele ser por invasión directa y los síntomas son anorexia, pérdida de peso, náuseas, vómitos, sangrado digestivo oculto y rara vez sangrado activo $^{1,3-6}$. En nuestra paciente destacaban la importante anemización y la hemorragia digestiva en forma de melenas, sin presentar inestabilidad hemodinámica que requiriese realizar una intervención de urgencia.

Endoscópicamente se presentan como múltiples ulceraciones mucosas o bien como una masa única ulcerada, similar a otro tipo de tumores con los cuales debe hacerse un diagnóstico diferencial. Dentro de los diagnósticos hay que considerar el adenocarcinoma, los GIST, los tumores neuroendocrinos, los linfomas (en particular el linfoma MALT [mucosa associated lymphoid tissue]) y la amiloidosis gastrointestinal, por lo que la realización de biopsias para el estudio anatomopatológico e inmunohistoquímico es crucial ${ }^{3,4,7}$. Desde el punto de vista inmunológico, un $27 \%$ expresan $\lg G$, un $33 \% \lg A$ y un $40 \% \lg \mathrm{M}^{1}$. En cuanto a su estudio histológico, es importante diferenciarlo del linfoma de células B de bajo grado asociado a las mucosas (linfoma MALT), ya que el estómago es su localización más habitual1,4.

Cuando se manifiesta como plasmocitoma solitario, tanto la cirugía o exéresis endoscópica como la radioterapia se han descrito como opciones terapéuti$\operatorname{cas}^{3,6,7}$. En ocasiones, cuando no es posible conseguir márgenes libres de tumor, se puede asociar radioterapia. La única excepción serían los plasmocitomas solitarios localizados en la cabeza y el cuello. En estos casos, la radioterapia es el tratamiento de elección ${ }^{6}$. Cuando se manifiesta asociado a mieloma múltiple se han descrito diversas opciones terapéuticas según su forma de presentación. Si se presentan en forma de hemorragia digestiva se puede realizar una endoscopia terapéutica (habitualmente con malos resultados y resangrado por tratarse de un tejido tumoral) o radioterapia local. Siddique, et al. ${ }^{8}$ reportan un caso de plasmocitoma duodenal tratado mediante embolización de la arteria gastroduodenal en un paciente con hemorragia digestiva recurrente y alto riesgo quirúrgico. La cirugía se suele indicar en los pacientes que presentan un sangrado incontrolable 0 recurrente, como en nuestro caso, y en aquellos que presentan clínica obstructiva ${ }^{3}$. La quimioterapia con esquemas como bortezomib + dexametasona se suele reservar para los casos más avanzados; es bien tolerada, con pocos efectos adversos y consigue remisiones completas, además de evitar los efectos adversos de la radiación abdominal ${ }^{9}$.

En nuestro caso, las biopsias preoperatorias confirmaban el diagnóstico definitivo, pero su resultado no se obtuvo hasta después de la intervención. Nuestra paciente presentaba un sangrado persistente, por lo que la cirugía resectiva estaba indicada. El conocimiento de la naturaleza de la lesión preoperatoriamente podría haber modificado la técnica quirúrgica y haber realizado un procedimiento menos agresivo, por lo que creemos importante realizar un diagnóstico histológico.

\section{Conflicto de intereses}

Los autores declaran que no existe conflicto de intereses.

\section{Financiamiento}

Los autores declaran que no existen fuentes de financiación. 


\section{Responsabilidades éticas}

Protección de personas y animales. Los autores declaran que para esta investigación no se han realizado experimentos en seres humanos ni en animales.

Confidencialidad de los datos. Los autores declaran que han seguido los protocolos de su centro de trabajo sobre la publicación de datos de pacientes.

Derecho a la privacidad y consentimiento informado. Los autores han obtenido el consentimiento informado de los pacientes y/o sujetos referidos en el artículo. Este documento obra en poder del autor de correspondencia.

\section{Bibliografía}

1. Chim CS, Wong WM, Nicholls J, Chung LP, Liang R. Extramedullary sites of involvement in hematologic malignancies: case 3. Hemorrhagic gastric plasmacytoma as the primary presentation in multiple myeloma. $\mathrm{J}$ Clin Oncol. 2002;20:344-7.
2. Talamo G, Cavallo F, Zangari M, Barlogie B, Lee CK, Pineda-Roman M, et al. Clinical and biological features of multiple myeloma involving the gastrointestinal system. Haematologica. 2006;91:964-7.

3. Telakis E, Tsironi E, Tavoularis G, Papatheodorou K, Tzaida O, Nikolaou A. Gastrointestinal involvement in a patient with multiple myeloma: a case report. Ann Gastroenterol. 2009;22:287-90.

4. Vicuña Arregui M, Borobio Aguilar E, Vila Costas JJ, Cruz Viguria Alegría M, Arrechea Irigoyen $\mathrm{M}$, Borda Celaya $\mathrm{F}$, et al. Plasmocitoma gástrico como causa infrecuente de hemorragia digestiva alta. Gastroenterol Hepatol. 2008;31:217-20.

5. Comba IY, Torres Luna NE, Cooper C, Crespo MW, Carilli A. A rare case of extramedullary plasmacytoma presenting as massive upper gastrointestinal bleeding. Cureus. 2019;11:e3993.

6. Honkisz BJ. Multiple myeloma with the primary gastric manifestation. $J$ Clin Case Rep. 2015;05(04). (COnsultado el 24 de marzo de 2020.) Disponible en: http://www.omicsgroup.org/journals/multiple-myeloma-with-the-primary-gastric-manifestation-2165-7920-1000525. php?aid $=55637$

7. Park CH, Lee SM, Kim TO, Kim DU, Jung WJ, Kim GH, et al. Treatment of solitary extramedullary plasmacytoma of the stomach with endoscopic submucosal dissection. Gut Liver. 2009;3:334-7.

8. Siddique I, Papadakis KA, Weber DM, Glober G. Recurrent bleeding from a duodenal plasmacytoma treated successfully with embolization of the gastroduodenal artery. Am J Gastroenterol. 1999;94:1691-2.

9. Katodritou E, Kartsios C, Gastari V, Verrou E, Mihou D, Banti A, et al. Successful treatment of extramedullary gastric plasmacytoma with the combination of bortezomib and dexamethasone: first reported case. Leuk Res. 2008;32:339-41. 